\title{
Analysis of the number of publications by Canadian anesthesiology departments from 2000-2013
}

\author{
Darren K. M. Lam, BSc • Ban C. H. Tsui, MD
}

Received: 24 March 2015/Accepted: 6 April 2015/Published online: 17 April 2015

(c) Canadian Anesthesiologists' Society 2015

\section{To the Editor,}

We previously performed an analysis of the academic output of anesthesiology departments across Canada from 2000-2004. ${ }^{1}$ Despite a steady increase in the total number of publications over this period, we identified a potentially concerning decrease in the number of randomized controlled trials (RCTs). Herein, we describe a follow-up analysis of publications by Canadian anesthesiology departments from 2005-2013 to evaluate whether the identified decrease in RCTs was a continuing trend.

The search criteria were the same as those described for our previous analysis ${ }^{1}$ and included all publications (i.e., all publications with a digital object identifier from January 1, 2000 to December 31, 2013). All articles with a printed publication date were included in our analysis. The Pearson correlation coefficient ( $r$ value) was used to analyze overall publication trends among Canadian university anesthesiology departments. Average annual percent change and annual percent change (APC) ${ }^{2}$ were calculated using the Joinpoint Regression Program 4.0.4 (Statistical Research and Applications Branch, National Cancer Institute, USA). ${ }^{3}$

Our search identified 3,084 eligible articles, and we included 2,947 of these for analysis. We found a steady increase in both the total number of articles published from 2005-2013 ( $r=0.86$; 95\% confidence interval [CI], 0.85 to 0.87 ) and the total number of publications from 2000-2013 $(r=0.91 ; 95 \% \quad \mathrm{CI}, 0.90$ to 0.92$)$ (Figure A). Using Joinpoint, APC analysis showed an average annual increase of $5.2 \%$ (95\% CI, 3.8 to 6.5 ) for all publications from 2000-2013. A small but significant increase in total

D. K. M. Lam, BSc · B. C. H. Tsui, MD ( $\varangle)$

Department of Anesthesiology \& Pain Medicine, University of Alberta, Edmonton, AB, Canada

e-mail: btsui@ualberta.ca
RCT publications occurred from 2005-2013 ( $r=0.17$; 95\% CI, 0.06 to 0.28 ), contrasting with the decrease that was observed from 2000-2004 $(r=-0.91)$. Our results show a slight overall increase in RCT publications throughout the entire period 2000-2013 ( $r=0.26$; 95\% CI, 0.17 to 0.35) (Figure B). Analysis of APC with Joinpoint over this period showed an annual increase of $1.0 \%$ (95\% CI, -1.7 to 3.9 ) for RCTs.

Following the analysis of publications from 2005-2013, it was reassuring to see that the apparent declining trend in RCT publications did not continue beyond 2004. In fact, publication of RCTs showed a slight overall increase since 2000. This result is comparable with the findings of Pagel and Hudetz who studied the publication output of American anesthesiology departments. ${ }^{4}$ As shown in the Figure, the total number of publications and RCTs fluctuates from year to year, suggesting that the time frame covered in our original analysis may have been too small to provide an accurate depiction of publication trends.

It is also reassuring that total publication activity increased in our latest period of analysis. A recent report by the Canadian Association of University Teachers ${ }^{5}$ reveals that the budget for the Canadian Institutes of Health Research, from which many medical researchers obtain their funds, fell 7.5\% from 2007-2013. Although this finding is not indicative of budgetary decreases for other granting agencies, it does reflect the economic climate with regard to research funding in Canada over the last decade. Our findings suggest that lack of funding was not a major impediment to performing anesthesiology-related research during this period.

As with the previous analysis, ${ }^{1}$ this study suffers from the possibility that institutions involved in multicentre or multidepartmental studies may be underrepresented since only the corresponding author's institution was used in the search. The use of PubMed to perform our search likely 
Figure Yearly total number of publications (A) and randomized controlled trials (RCTs) only (B) for the period 2000-2013. Trendlines show the overall increase in the total number of publications and RCTs over the period $2000-2013 ; r$ values $(95 \%$ confidence intervals $[\mathrm{CI}])$ are shown for the entire period
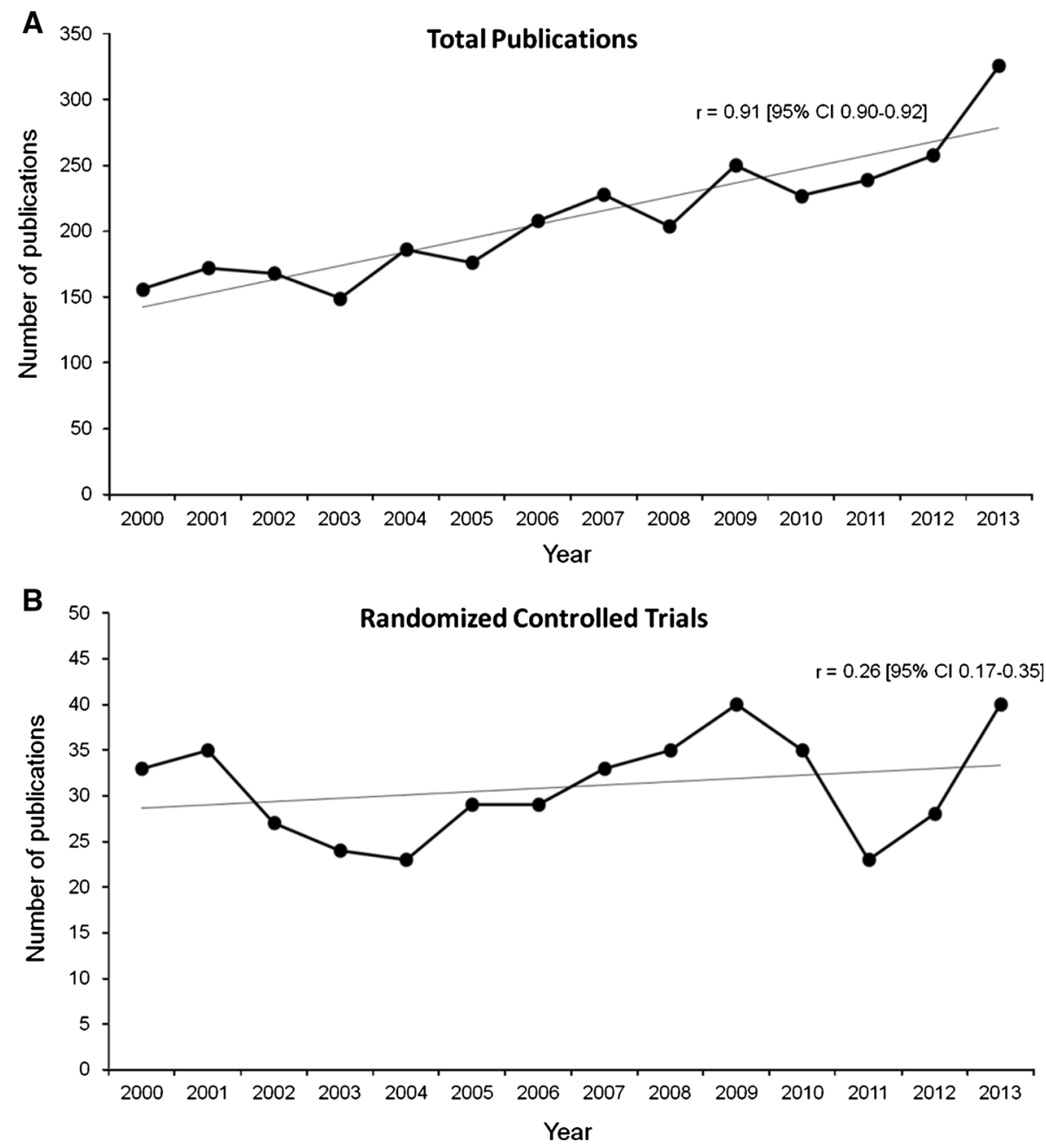

resulted in some eligible publications being overlooked. Occasionally, an article will be listed in PubMed for which the corresponding author's affiliation cannot be searched. In these rare cases, our search would not have captured articles whose corresponding author was affiliated with a Canadian anesthesiology department. Our current analysis is also limited to assessing publication quantity; therefore, any assessment of publication quality - which is arguably a subjective concept - could not be done.

In summary, we have shown that the declining trend in the publication of RCTs among Canadian anesthesiology departments did not continue beyond 2004. On the contrary, we show that, in the period 2000-2013, overall publication activity increased steadily, a reassuring trend in light of declining federal research funding during the latter part of the period.

Acknowledgements The authors thank Ian Armstrong, Natalie Chua, Jason Ha, Sarah Henschke, Kristyn Kurio, Danika Leung, Emilie Robertson, and Cam Shillington for their assistance with the categorization of the research articles. The authors also thank Dr. Gareth Corry for his assistance with the manuscript.
Conflicts of interest None declared.

Financial disclosures Dr. Tsui is supported in part by a Clinical Scholar Award from the Alberta Heritage Foundation for Medical Research (AHFMR) and a CAS/Abbott Laboratories Career Scientist Award from the Canadian Anesthesiologists' Society. Darren Lam was supported by the Canadian Institutes of Health Research (CIHR) Health Professional Student Research Award.

\section{References}

1. Tsui BC, Li LX, Ma V, Wagner AM, Finucane BT. Declining randomized clinical trials from Canadian anesthesia departments? Can J Anesth 2006; 53: 226-35.

2. Clegg LX, Hankey BF, Tiwari R, Feuer EJ, Edwards BK. Estimating average annual per cent change in trend analysis. Stat Med 2009; 28: 3670-82.

3. Kim HJ, Fay MP, Feuer EJ, Midthune DN. Permutation tests for joinpoint regression with applications to cancer rates. Stat Med 2000; 19: 335-51.

4. Pagel PS, Hudetz JA. Recent trends in publication of basic science and clinical research by United States investigators in anesthesia journals. BMC Anesthesiol 2012; 12: 5.

5. Canadian Association of University Teachers. Federal Funding of Basic Research. CAUT Education Review 2013; 13: 1-6. 